\title{
A role for low-abundance miRNAs in colon cancer: the miR-206/Krüppel-like factor 4 (KLF4) axis
}

\author{
Mansi A Parasramka', W Mohaiza Dashwood ${ }^{1}$, Rong Wang ${ }^{1}$, Hassaan H Saeed ${ }^{1}$, David E Williams ${ }^{1,2}$, Emily Ho ${ }^{1,3}$ \\ and Roderick H Dashwood ${ }^{1,2,4^{*}}$
}

\begin{abstract}
Background: MicroRNAs (miRNAs or miRs) are short non-coding RNAs that affect the expression of genes involved in normal physiology, but that also become dysregulated in cancer development. In the latter context, studies to date have focused on high-abundance miRNAs and their targets. We hypothesized that among the pool of low-abundance miRNAs are some with the potential to impact crucial oncogenic signaling networks in colon cancer.

Results: Unbiased screening of over 650 miRNAs identified miR-206, a low-abundance miRNA, as the most significantly altered miRNA in carcinogen-induced rat colon tumors. Computational modeling highlighted the stem-cell marker Krüppel-like factor 4 (KLF4) as a potential target of miR-206. In a panel of primary human colon cancers, target validation at the mRNA and protein level confirmed a significant inverse relationship between miR-206 and KLF4, which was further supported by miR-206 knockdown and ectopic upregulation in human colon cancer cells. Forced expression of miR-206 resulted in significantly increased cell proliferation kinetics, as revealed by real-time monitoring using HCT116 cells.
\end{abstract}

Conclusions: Evolutionarily conserved high-abundance miRNAs are becoming established as key players in the etiology of human cancers. However, low-abundance miRNAs, such as miR-206, are often among the most significantly upregulated miRNAs relative to their expression in normal non-transformed tissues. Low-abundance miRNAs are worthy of further investigation, because their targets include KLF4 and other pluripotency and cancer stem-cell factors.

Keywords: Cancer stem cells, Colon cancer, Epigenetics, KLF4, microRNAs, miR-206, Pluripotency factors

\section{Background}

MicroRNAs (miRNAs or miRs) influence multiple stages of cancer development, via post-transcriptional mechanisms that degrade or repress target messenger RNAs (mRNAs) [1]. Several miRNAs with critical roles in early embryonic development [2] become aberrantly expressed in tumors [3]. For example, miR-21 is a high-abundance miRNA upregulated in cancers of the breast, lung, colon, liver, pancreas, prostate, esophagus, brain, and thyroid; targets of miR-21 include phosphatase and tensin homolog, tropomyosin 1 , and programmed cell death $4[4,5]$.

\footnotetext{
* Correspondence: rod.dashwood@oregonstate.edu

'Linus Pauling Institute, Oregon State University, Corvallis, Oregon, USA ${ }^{2}$ Department of Environmental and Molecular Toxicology, Oregon State University, Corvallis, Oregon, USA

Full list of author information is available at the end of the article
}

Evolutionarily conserved high-abundance miRNAs, such as miR-21, have been profiled in various human cancers [6-8], but little is known about the role of low-abundance miRNAs. We hypothesized that certain low-abundance miRNAs might regulate key players in normal physiology such that, under normal circumstances, their expression is tightly restricted.

One such candidate is miR-206. This miRNA has been implicated in breast and lung cancer via the inhibition of notch3 signaling, cell migration, proliferation, metastasis, and invasion [9,10]. Moreover, levels of miR-206 were inversely proportional to c-met expression, an important proto-oncogene in rhabdomyosarcoma [11]. Lin et al. [12] identified an autoregulatory feedback loop between miR-206 and Krüppel-like factor 4 (KLF4). This zinc finger protein plays a crucial role in early

\section{Biomed Central}


development and cancer stem-cell biology [13]. KLF4 promotes tumor formation in tissues such as breast and skin $[14,15]$, but suppresses malignancy in cervix, prostate, bladder, esophagus, and colon [16-21]. Loss of the KLF4 region on chromosome 9q is reported in $25 \%$ to $50 \%$ of sporadic colorectal cancers, and a significant decrease in KLF4 expression is observed in adenomas and adenocarcinomas of the large and small intestine [22,23].

In a recent investigation of carcinogen-induced rat colon tumors [24], we identified a loss of multiple let-7 family members coinciding with increased expression of miRNA-binding proteins Lin28A/Lin28B, as well as the stem-cell factors c-Myc, Sox2, Oct-3/4, and NANOG. One notable omission from the list of downstream targets was Klf4, a well-established pluripotency factor [13]. Interestingly, among the entire set of 679 miRNAs profiled in rat colon tumors, the greatest relative increase compared with normal tissue was for the low-abundance
miR-206. This investigation, therefore, sought to clarify the role of miR-206 and its putative target KLF4 in colon cancer development. This report provides the first comprehensive analysis of the miR-206/KLF4 axis in parallel studies involving a preclinical colon carcinogenesis model, human primary colon cancers, and a panel of human colon cancer cells.

\section{Results}

Increased miR-206 and attenuated Klf4 expression in rat colon tumors

Among 679 unique miRNAs profiled, miR-206 was the most dramatically altered miRNA in rat colon tumors, exhibiting $\sim 73$-fold higher expression relative to the corresponding normal-looking colonic mucosa (microarray data not shown). Metacore pathway analysis predicted multiple targets of miR-206, including KLF4 (Figure 1A), which was further supported by sequence

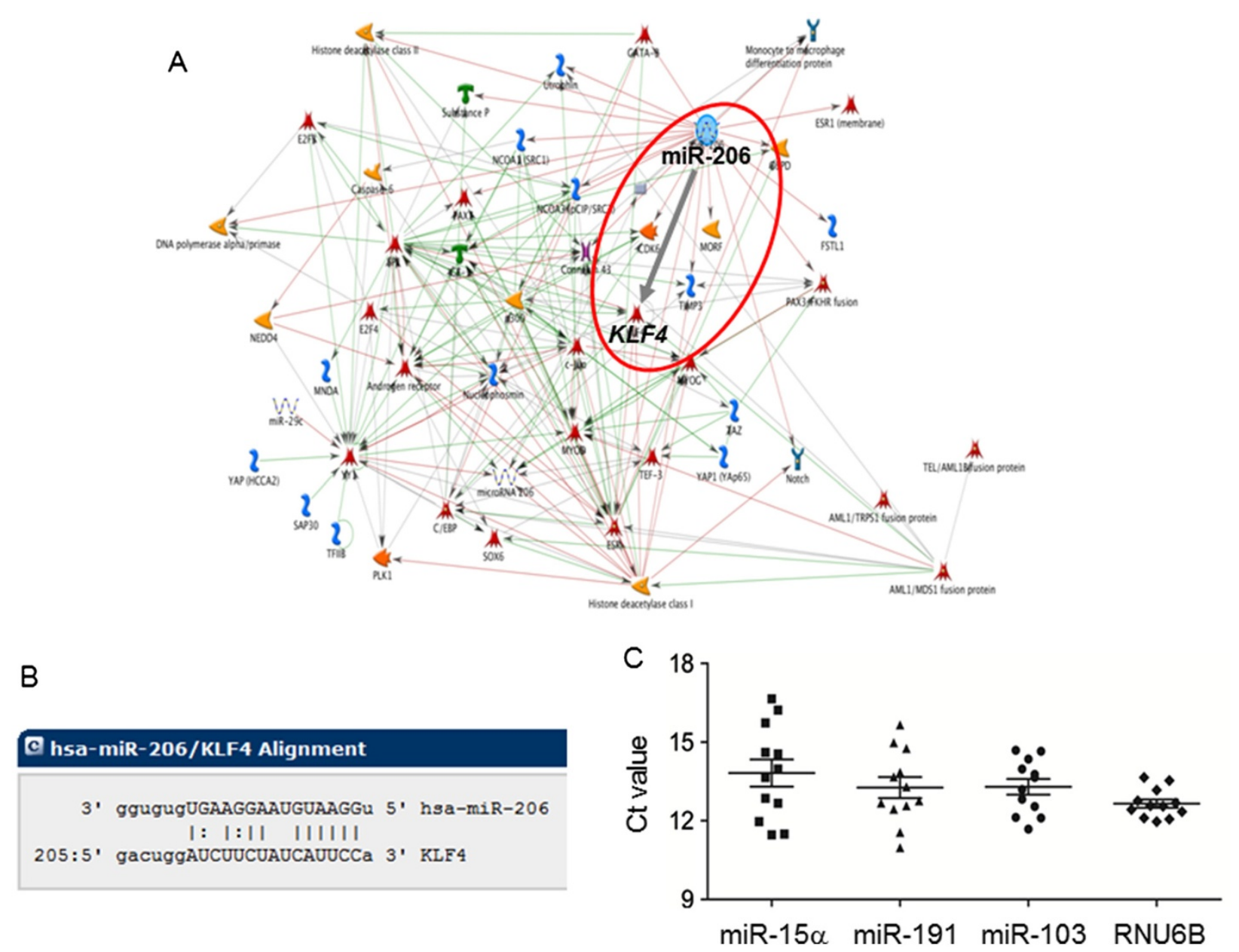

Figure 1 The miR-206/KLF4 axis in colon cancer. MicroRNA arrays [22] identified miR-206 as a low-abundance miRNA with potentially important roles in carcinogen-induced rat colon tumors. (A) Metacore pathway analysis encompassing rat, mouse, and human miRNAs highlighted KLF4 as an important target of miR-206, which was supported (B) by computational alignment. (C) Quantitative real-time PCR (qRTPCR) assays revealed $R N U 6 B$ as a suitable internal control for subsequent experiments using the rat model, see Figure 2. 
complementarity alignment (Figure 1B). To validate these findings, we first screened a number of potential endogenous controls, selecting RNU6B as a suitable candidate for subsequent quantitative real-time polymerase chain reaction (qRT-PCR) assays (Figure 1C).

In six randomly selected rat colon tumors, miR-206 levels were expressed at higher levels, relative to the corresponding normal-looking colonic mucosa (Figure 2A). These colon tumors had the predicted inverse relationship with Klf4 mRNA levels, normalized to Gapdh (Figure 2B). Specifically, for miR-206 the tumor mean \pm SD was $1.8 \times 10^{-5} \pm 4.5 \times 10^{-7}$ versus matched controls $2.8 \times 10^{-6} \pm 4.6 \times 10^{-6} \quad(P<0.0001, n=6)$. For Klf4 the corresponding data were $0.084 \pm 0.0075$ in tumors versus
$0.25 \pm 0.034$ in normal colon $(P<0.001, n=6)$. However, we did note that one colon tumor with a high relative level of miR-206 did not exhibit a correspondingly reduced level of Klf4 (compare data in Figures 2A and 2B for Case 3). These data supported a possible role for miR-206 regulating Klf4 in some but not all rat colon tumors.

\section{An inverse relationship between miR-206 and KLF4 in human primary colon cancers}

Based on a prior report [25], human primary colon cancers were first screened for a suitable endogenous control (data not shown); miR-191 was selected for subsequent qRT-PCR analyses. There was an inverse
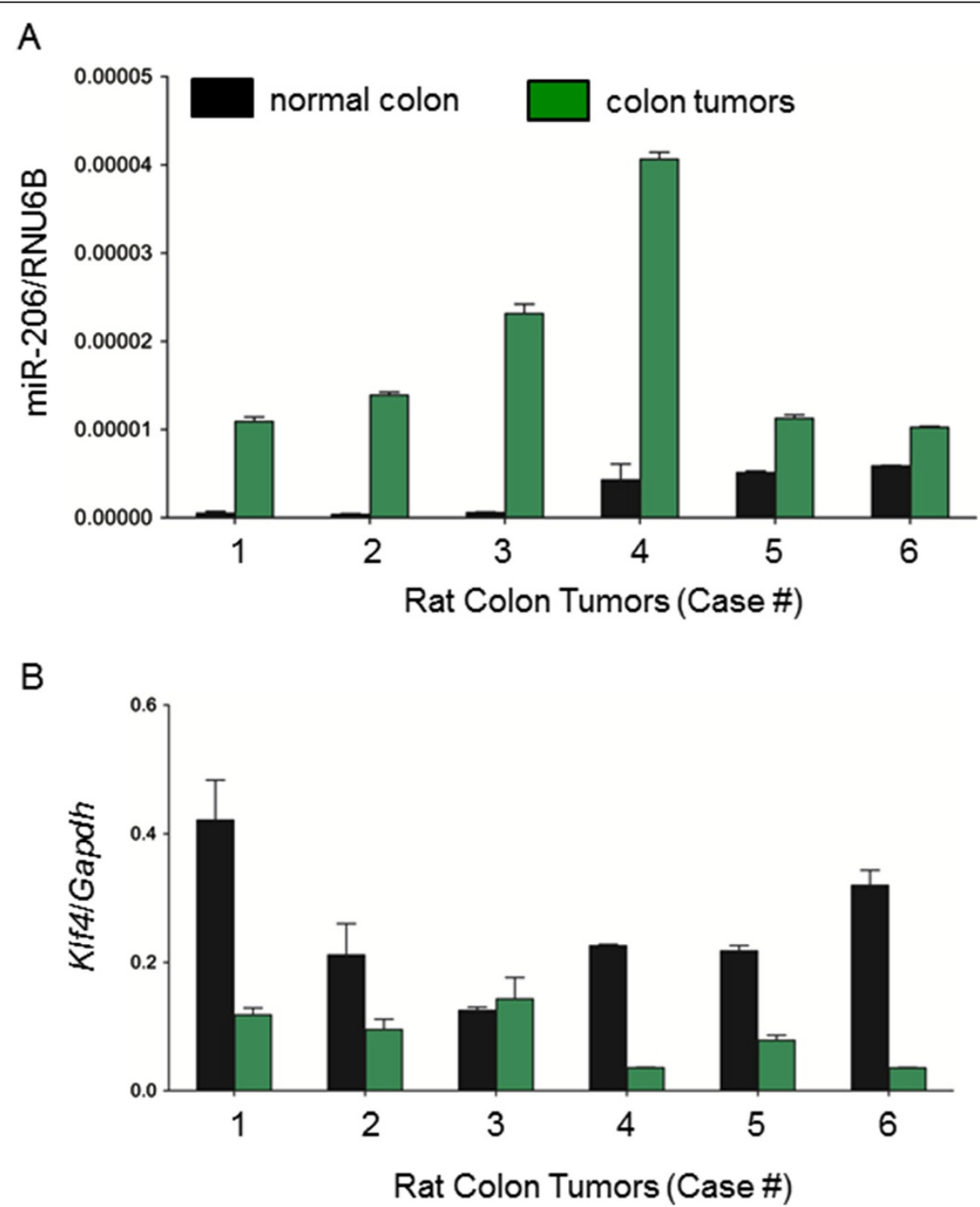

Figure 2 miR-206 and Klf4 expression in rat colon tumors. Carcinogen-induced colon tumors and adjacent normal-looking colonic mucosa from a prior study [22] were examined for (A) miR-206 expression, normalized to RNU6B, and (B) KIf4 mRNA expression normalized to Gapdh. For the six colon tumors shown, data bars indicate mean \pm SD, $n=3$. 
association between miR-206 levels and KLF4 mRNA expression among the 21 human primary colon cancers examined (Figure 3A, inset, $r^{2}=0.525, P<0.05$ ). Several of the clinical cases had higher miR-206 in the cancer compared with the patient-matched control (Figure 3B, green versus black bars, respectively); see for example Cases 1, 5, 7, 12, 14, 17, 18, and 21. However, the reverse situation was noted in Cases 6, 8, 9, and 15 (Figure 3B).

Upon further analysis, low KLF4 mRNA levels were detected in most colon cancers, with the exception of Cases 3 and Case 6 (Figure 3C, normalized to GAPDH). Western immunoblotting confirmed the attenuated KLF4 protein level in most, but not all, tumors $(\mathrm{T})$ compared with patient-matched normal $(\mathrm{N})$ tissues (Figure $3 \mathrm{C}$, $\beta$-actin loading control). As noted in the rat model, the inverse association between miR-206 and KLF4 was not always apparent; for example, Case 9 exhibited low relative expression of both miR-206 and KLF4 in the cancer compared with the patient-matched control (Figure 3B,C). However, inspection of the data in Figures $3 \mathrm{~B}$ and $3 \mathrm{C}$ supported the general hypothesis in 12/21 (57\%) of the human primary colon cancers. For miR-206, the tumor mean $\pm \mathrm{SD}$ was $1.8 \times 10^{-4} \pm 1.6 \times 10^{-5}$ versus $1.3 \times 10^{-4} \pm 4.6 \times 10^{-6}$ in matched controls $(P<0.05)$. For KLF4, the corresponding data were $0.68 \pm 0.0746$ in tumors versus $1.467 \pm 0.194$ in controls $(P<0.01)$.

\section{Knockdown or ectopic upregulation of miR-206 alters KLF4 levels in human colon cancer cells}

In an initial screen of human colon cancer cell lines (Figure 4), SW48 cells had the highest constitutive levels of miR-206 and low relative expression of KLF4, whereas SW480 cells exhibited the highest endogenous KLF4 levels and low miR-206 expression. For the same molecular endpoints, intermediate expression was detected in HCT116 and HT29 cells. Caco-2 cells exhibited low endogenous levels of both miR-206 and KLF4, similar to the non-cancer colon embryonic epithelial cell line CCD841 (Figure 4, solid black bar).

We elected to use an intermediate expressing cell line, HCT116, for subsequent experiments, designed to knockdown or ectopically upregulate miR-206 (Figure 5). A dose-dependent increase in miR-206 expression was observed on transfecting cells with miR-206 mimic (30 to $70 \mathrm{nM}$ ), relative to untreated cells (black bar), vehicle treatment (30 and $40 \mu \mathrm{l}$ HiPerFectamine, blue bars), or sham control (green bars). Under the same conditions, enforced increase of miR-206 resulted in loss of KLF4 (compare orange bars in Figure $5 \mathrm{~B}$ with those in Figure 5A). In contrast, HCT116 cells treated with miR206 inhibitor had a significant reduction in miR-206 expression, and this was associated with a corresponding increase in KLF4 (compare pink bars in Figure 5A and
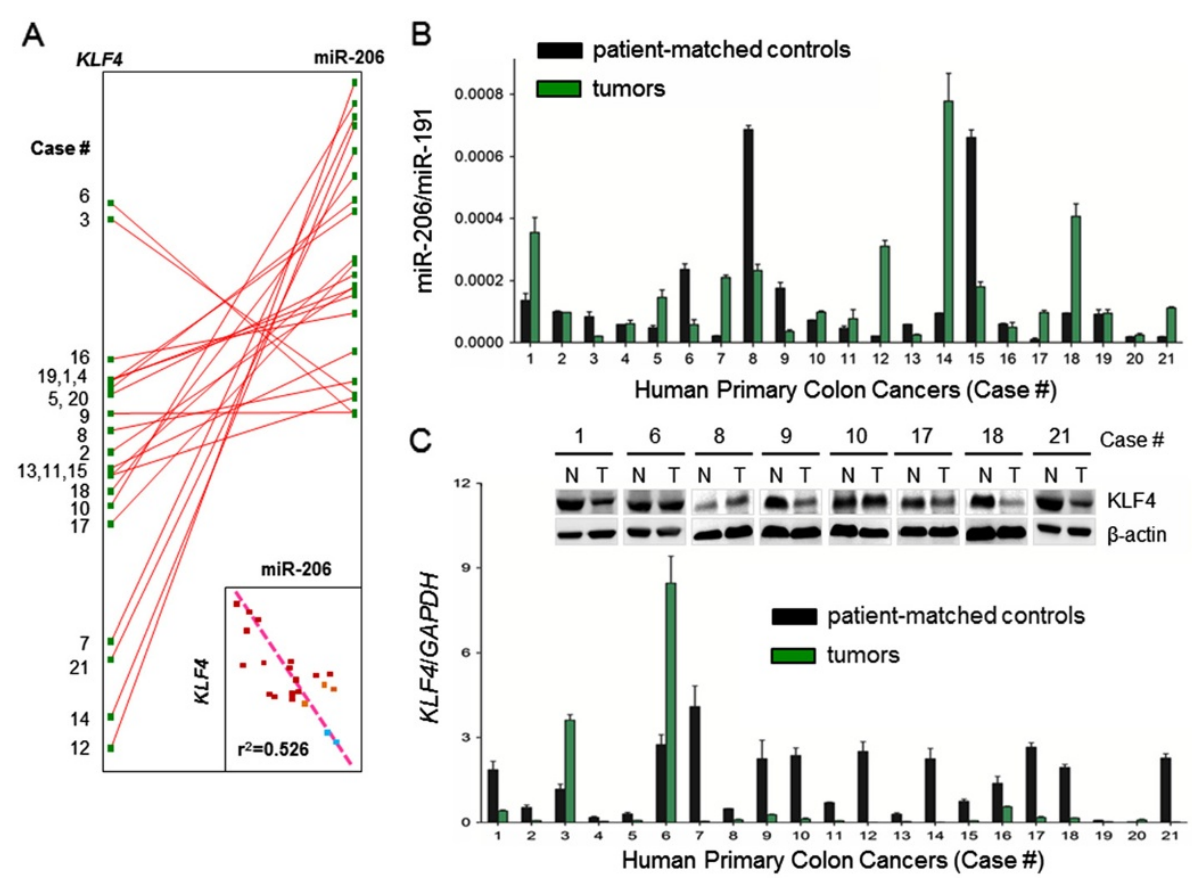

Figure 3 Inverse association between miR-206 and KLF4 in human colon cancers. (A) Twenty-one human primary colon cancers were examined for KLF4 mRNA expression normalized to GAPDH, and for miR-206 normalized to miR-191. Inset: the inverse correlation was significant, $r^{2}=0.525, P<0.05$. (B) Expression of miR-206/miR-191 in 21 primary colon cancers and their patient-matched controls. (C) Expression of KLF4/ GAPDH mRNA in the same tumors and matched controls. Representative Western immunoblots are also shown for KLF4 protein normalized to $\beta$-actin. 


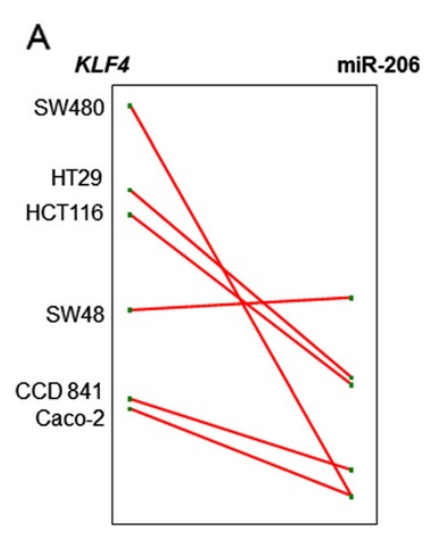

\section{B}
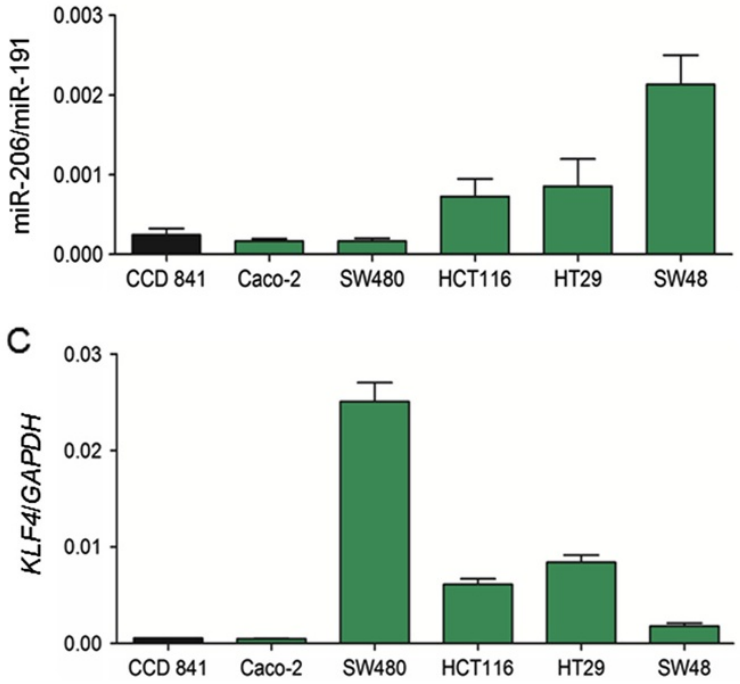

Figure 4 Constitutive expression of miR-206 and KLF4 in human colon cancer cells. (A) Inverse trends for KLF4 and miR-206 in five colon cancer cell lines and non-transformed colonic epithelial CCD841 cells. (B) miR-206/miR-191 and (C) KLF4/GAPDH levels in five colon cancer cell lines (green bars) and CCD841 cells (black bars); data bars indicate mean \pm SD, $n=3$.
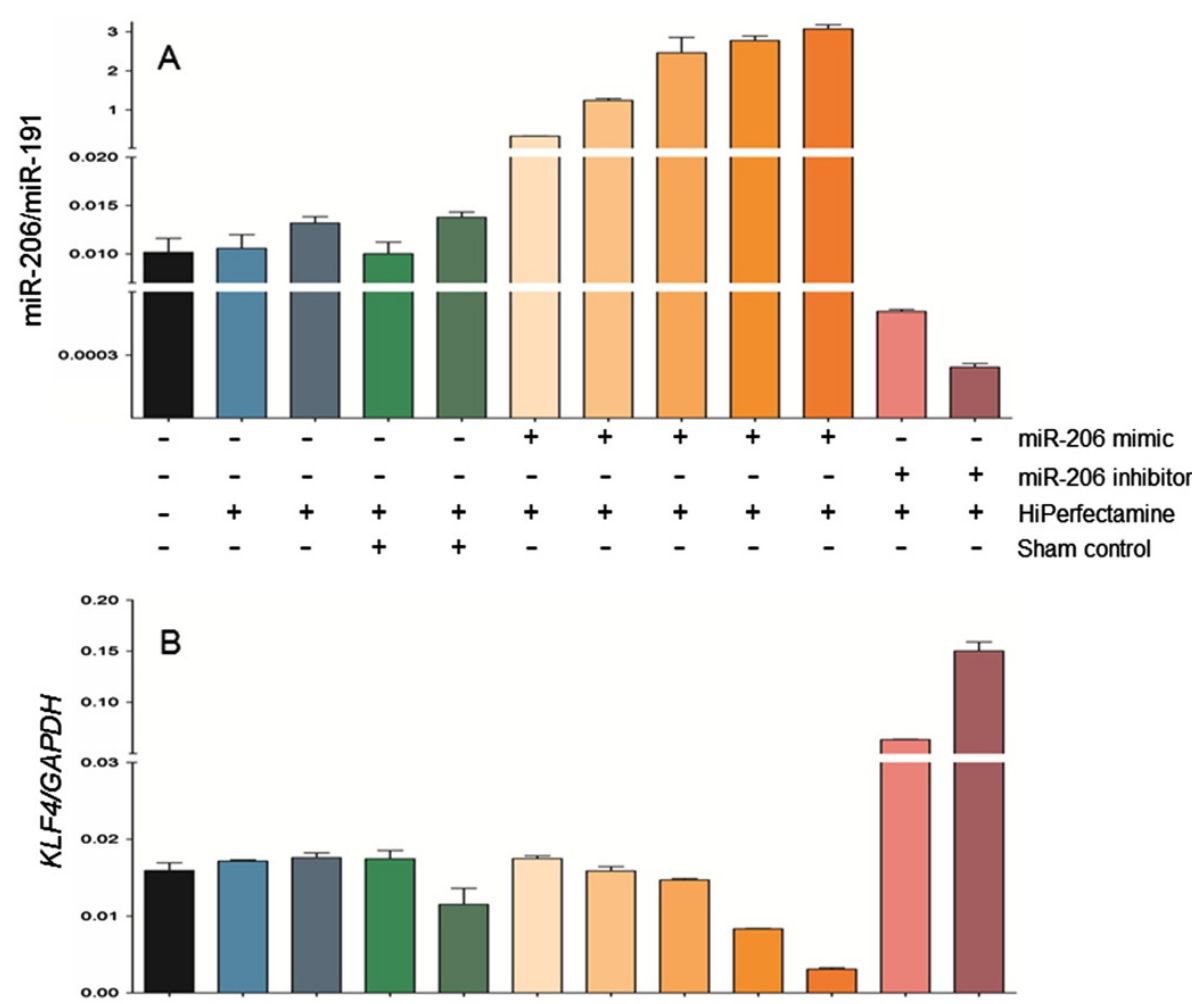

Figure 5 Knockdown and ectopic upregulation of miR-206 reciprocally regulates KLF4. Human HCT116 colon cancer cells were transfected with increasing concentrations of a miR-206 mimic (graded orange-shaded bars indicating increasing miR-206), or miR-206 inhibitor (pink bars). Black, blue, and green bars designate the various controls, as mentioned in the text. (A) miR-206/miR-191 expression and (B) KLF4/GAPDH levels were measured as described for human primary colon cancers, see Figure 3 legend. Data bars = mean $\pm S D, n=3$. 
$5 \mathrm{~B})$. These findings supported an inverse association between miR-206 and KLF4 in colon cancer.

\section{Enforced increase of miR-206 levels augments} proliferation kinetics in human colon cancer cells

Eight hours after HCT116 cells were transfected with miR-206 mimic, qRT-PCR assays corroborated the expected increase in miR-206 levels relative to vehicle and sham controls (Figure 6A,B). Reduced KLF4 expression was also confirmed under the same experimental conditions (data not shown). Cells were loaded onto E-plates (xCELLigence system, Roche) for real-time monitoring assays [26], which revealed a significant increase in cell proliferation kinetics following the enforced increase of miR-206 levels (Figure 6C, inset, $P<0.05$ ).

\section{Discussion}

The KLF4 protein contains both activator and repressor domains, and can function as a positive or negative regulator of gene expression [27-29]. Oncogenic effects of KLF4 have been reported in breast, skin, and lung cancers $[14,15,30]$. However, KLF4 also acts as a tumor suppressor in human colon cancer development [31]. Information on tumor stage was available for some, but not all, of the human primary colon cancers examined here, and as a consequence we could not corroborate an early report suggesting KLF4 as a prognostic predictor of colon cancer [20]. Prior studies in human colon cancer cell lines and primary colorectal cancers implicated hypermethylation of the KLF4 gene promoter, loss of heterozygosity, or mutation of the open reading frame [17]. Recently, a KLF4/miR-206 autoregulatory feedback loop was reported to regulate protein translation reciprocally in normal and cancer cells [12]. In this investigation of rat colon tumors, human primary colon cancers, and human colon cancer cell lines, the data supported an inverse trend between KLF4 and miR-206.

Located on chromosome 6p12.2, miR-206 is similar in expression and function to miR-1, but its sequence differs by four nucleotides [32]. Studies in breast cancer models have reported tumor suppressive effects of miR206 due to its pro-apoptotic properties, via the inhibition of notch3 signaling and cell migration $[9,33]$ or proliferation [10]. Furthermore, miR-206 was one of five miRNAs that exhibited stage-dependent differential expression in human colorectal cancers [33]. Interestingly, the latter report noted, in contrast to the current study, that miR-206 levels were more typically attenuated whereas let-7a was increased in the cancers. Given that let-7 family members are normally ascribed a tumor suppressor function, the authors speculated that a high let-7a/low miR-206 'signature' might designate colon tumors with a unique phenotype in terms of cancer progression, compartmentalization, or microenvironment [33]. This subset of cancers would differ from the more typical scenario involving increased miR-206 and reduced let-7a expression.

Loss of let-7a, and of other let-7 family members, coincided with changes in other high-abundance miRNAs in the heterocyclic amine-induced rat colon tumors examined here [24]. Interestingly, however, when the dataset of 679 miRNAs was taken in its entirety, the low-abundance miR-206 was identified as the most significantly upregulated (up to 73-fold) in rat colon tumors relative to normal colonic mucosa. In a separate study using azoxymethane as the initiating agent, miR206 was also significantly upregulated ( 100-fold) in rat colon tumors [34]. The latter report did not pursue

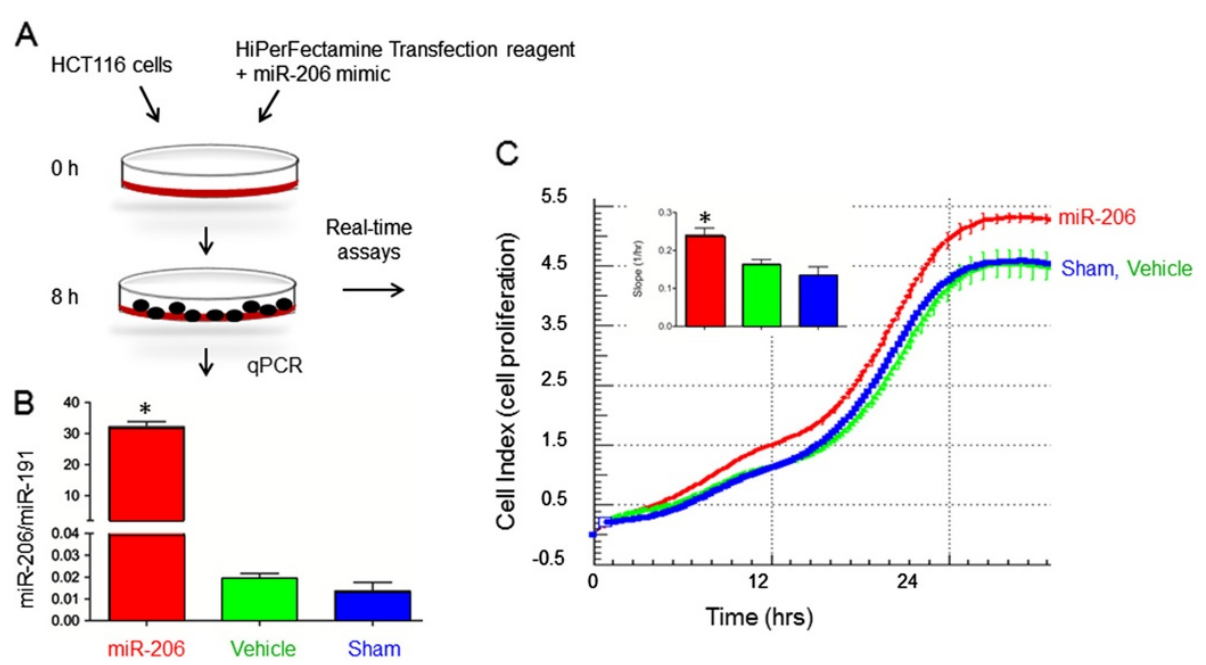

Figure 6 Cell proliferation is increased by miR-206. (A) Experimental protocol for the treatment of HCT116 cells. (B) qRT-PCR data confirmed upregulation of miR-206 expression. (C) Real-time monitoring assays using the xCELLigence system [26]. Data bars indicate mean $\pm S D, n=3$. 
miR-206 further, owing to its low abundance relative to other miRNAs. Nonetheless, we were intrigued that two quite different colon carcinogens increased miR-206 so dramatically in colon tumors, relative to normal colonic mucosa.

Thus, we next examined a panel of human primary colon cancers and detected increased miR-206 levels in $\sim 50 \%$ of the cases. As predicted by computational modeling, a significant inverse relationship was noted between miR-206 and KLF4 (Figure 3A, inset). Other known colon cancer miRNA signatures were confirmed, such as an increase in miR-21 and a decrease in miR-98, but no concordance was observed for these miRNAs and KLF4 (data not shown). To provide further proofof-concept, we examined a panel of human colon cancer cell lines and noted that SW480 and SW48 cells provided the best evidence for an inverse association between miR-206 and KLF4. In cells with intermediate constitutive levels, forced expression or knockdown of miR-206 resulted in the expected reciprocal changes in KLF4, and miR-206 ectopic upregulation increased cell proliferation kinetics in real-time monitoring assays. Interestingly, Caco- 2 cells resembled non-transformed CCD841 cells, as well as certain human primary colon cancers (Cases 9 and 15, Figure 3), in having low expression levels of both miR-206 and KLF4. The latter signature appeared to involve slower cell proliferation and doubling time, although further work is needed to clarify this possibility, especially in vivo. Among the other factors that might contribute in vitro, miR-206 expression was influenced by cell confluency, being about threefold higher at $70 \%$ versus $20 \%$ confluency (MAP et al., unpublished data).

In contrast to these observations, cancers of the breast and lung have been characterized as exhibiting low miR206 expression levels, suggesting a possible tumor suppressor function $[9,10,12]$. Changes in the expression of KLF4 also can be site specific, being upregulated in cancers of the breast, skin, and lung, but attenuated in colon and gastric tumors $[14,15,19,31]$. The prior investigation of high-abundance miRNAs in rat colon tumors highlighted a role for c-Myc, Oct-3/4, and Sox2 [24], whereas the present work has implicated a fourth 'defined factor' for pluripotency, namely KLF4 [13]. Further studies are in progress on the role of pluripotency factors, cancer stem markers, and other potential molecular targets of the miR-206/KLF4 axis (Figure 1).

\section{Conclusions}

This investigation has focused on the possible role of miR-206 and one of its predicted targets, KLF4, in colon cancer development. As a low-abundance miRNA, miR206 might have been overlooked but for the fact that it was the most significantly increased miRNA in rat colon tumors, relative to normal colonic mucosa. An inverse correlation was noted between miR-206 and KLF4 in a panel of human primary colon cancers, which was supported by experimental studies involving knockdown and ectopic upregulation of miR-206 levels in human colon cancer cells. We conclude that among the lowabundance miRNAs normally ignored or 'discarded' during profiling studies there may be key players worthy of mechanistic investigation, depending on the tissue and cancer type.

\section{Methods}

\section{Cell culture and transfection}

Human colorectal cancer lines HCT116, HT29, Caco2, SW48, and SW480 (American Type Culture Collection, Manassas, VA) were maintained in McCoy's 5A medium (Invitrogen) supplemented with $10 \%$ heat-inactivated fetal bovine serum (FBS, Hyclone Laboratories), 100 units $/ \mathrm{ml}$ penicillin, and $100 \mu \mathrm{g} / \mathrm{ml}$ streptomycin at $37^{\circ} \mathrm{C}$ in $5 \% \mathrm{CO}_{2}$. The human embryonal colon epithelial cell line CCD841 was maintained in Eagle's minimum essential medium (EMEM) according to the recommended ATCC protocol. Each cell line was confirmed independently to be of human origin with no mammalian interspecies contamination, and had the correct genetic profile based on allele-specific markers (Idexx Radil, Columbia, MO).

Transfection of HCT116 cells was performed using a miR-206 mimic (Qiagen, Valencia, CA, USA). Cells were seeded in 100-mm cell-culture grade Petri dishes and transfected at 70\% confluency using Qiagen HiPerFectamine Transfection reagent, according to the manufacturer's protocol. Final concentrations were as follows: miR-206 mimic 30-70 nM, miR-206 inhibitor 50 and 75 nM, AllStars Negative Control 25-75 nM, or HiPerFectamine 30-40 $\mu \mathrm{l}$ in $1000 \mu \mathrm{l}$ serum-free McCoy's 5A medium. Untreated cells received complete media only. Cells were harvested after $60 \mathrm{~h}$ for determination of miRNA and mRNA expression.

\section{Rat and human colon tumors}

The preclinical study was approved by the Institutional Animal Care and Use Committee (ACUP 3168). Male F344 rats, 3-4 weeks of age, were housed in a ventilated temperature-controlled room at $25^{\circ} \mathrm{C}$ with a $12 \mathrm{~h}$ light and dark cycle. After acclimatization to the basal diet (AIN-93 G), rats were treated by oral gavage with three cycles of a heterocyclic amine carcinogen. Details of the dosing protocol were reported elsewhere [24]. After the last dose of carcinogen, in week 18, rats were switched to AIN-93 $\mathrm{M}$ diet until the study was terminated at 52 weeks. Colon tumors and normal-looking colonic mucosa samples were flash-frozen in liquid nitrogen and stored at $-80^{\circ} \mathrm{C}$ prior to miRNA isolation. 
Primary human colon cancers and patient-matched controls were provided under an IRB-approved protocol by Steven F. Moss and Lelia Simao (Rhode Island Hospital, Providence, RI). The tumors were characterized as late-stage adenocarcinomas, as reported by Wang et al. [26].

\section{MicroRNA extraction}

Cells from experiments conducted in vitro were homogenized in ice-cold lysis buffer and flash-frozen. After thawing, cell lysates, human and rat colon tumors, and the corresponding matched controls were homogenized on ice and microRNA isolation was performed using the miRNeasy kit (Qiagen). RNA quality and integrity was evaluated from the absorbance at 260 and $280 \mathrm{~nm} \mathrm{(260/}$ 280 ratio $>1.9$ ).

\section{MicroRNA microarrays and computational analyses}

Microarray analyses of 679 unique mature target rat miRNAs (from miRBase version 17, http://miRBase.org) were performed by LC Sciences (Houston, TX), as detailed elsewhere [24]. Images were collected using a GenePix 4000B scanner (Molecular Devices, Sunnyvale, CA) and digitized using Array-Pro image analysis software (Media Cybernetics, Bethesda, MD). Data were analyzed by first subtracting the background and then normalizing the signal using a locally weighted regression filter. Cluster plots were generated using software from The Institute for Genomic Research.

For human primary colon cancers and their matched controls, miR-206 and KLF4 expression values were analyzed by Benjamini-Hochberg correction using ArrayStar software (DNASTAR, Inc., Madison, WI, USA). Scatter plots were evaluated by simple linear regression analysis. Interaction between the expression profiles among the samples was compared by hierarchical cluster analyses and line graph using ArrayStar software. A difference with $P<0.05$ was considered statistically significant.

\section{Biological network analyses}

Putative target mRNAs of miR-206 were examined via MetaCore pathway analysis (GeneGo Inc., St Joseph, MI, USA). For pathway enrichment analysis, $P$ values were calculated using the formula for hypergeometric distribution, reflecting the probability of a pathway arising by chance. Pathway maps were prioritized based on statistical significance.

\section{Validation of microRNA expression}

The qRT-PCR analyses used the Qiagen miScript kit according to the manufacturer's instructions, with miR206-specific primers. RNA $(\sim 1 \mu \mathrm{g})$ from colon tumors or normal controls was reverse-transcribed, and qRTPCR was performed in a $20 \mu \mathrm{l}$ reaction containing
miScript Universal primer, miRNA specific-miScript primers, SYBR green mix, and template cDNA. Reactions were conducted in triplicate and fluorescence intensities were acquired using a LightCycler 480 II (Roche Applied Science, Indianapolis, IN, USA). Relative miRNA expression was quantified by determining the point at which the fluorescence accumulation entered the exponential phase $(\mathrm{Ct})$, and the $\mathrm{Ct}$ ratio of the target was normalized to small nuclear RNA U6B (RNU6B) or miR-191.

\section{Messenger RNA expression analysis}

Target mRNAs were quantified by qRT-PCR and normalized to glyceraldehyde-3-phosphate dehydrogenase (human $G A P D H$, rat Gapdh). Briefly, $1 \mu \mathrm{g}$ total RNA was reversetranscribed using SuperScript III First Strand Synthesis Supermix Kit (Invitrogen, Eugene, OR, USA), and qRTPCR was conducted in a $20 \mu$ reaction containing cDNAs, SYBR Green I dye, and primers KLF4-F: CCAATTACC CATCCTTCCTG, KLF4-R: CGATCGTCTTCCCCTCTTTG (human) or Klf4-F: CAGACCTGGAAAGTGGTGG, Klf4-R: ACCTGTGTTGCCCGCAGCC (rat). Experiments were conducted in a Light Cycler $480 \mathrm{II}$, and the Ct ratio of the target gene to GAPDH or Gapdh was calculated. Three separate experiments were performed in triplicate, for each sample.

\section{Western immunoblotting}

KLF4 protein levels were examined using the Western immunoblotting methodology reported elsewhere, with $\beta$-actin as loading control [24]. The primary antibody was rabbit polyclonal KLF4 lot no. GR45275-3 (Abcam, Cambridge, MA, USA), at 1:500 dilution.

\section{Cell proliferation assays}

Real-time cell proliferation assays were conducted using the xCELLigence System (Roche). HCT116 human colon cancer cells were transfected with miR-206 mimic, as described previously. Cells transfected with mimic, vehicle (HiPerFectamine), or sham negative control were harvested at $8 \mathrm{~h}$. Cells $\left(1.5 \times 10^{3}\right)$ in $100 \mu \mathrm{l}$ serum-free media were loaded onto the pre-soaked Roche E-plates and measurements were taken at intervals of $10 \mathrm{~min}$ [26].

\section{Statistics}

Data were plotted as mean $\pm \mathrm{SD}$ and compared using one-way and two-way ANOVA (Graphpad Prism 5 software). In the figures, significant outcomes were shown as: ${ }^{*} P<0.05,{ }^{* *} P<0.01$, **** $P<0.001$.

\section{Abbreviations}

ANOVA, analysis of variance; EMEM, Eagle's minimum essential medium; KLF4, Krüppel-like factor 4; KLF4, Human gene coding for KLF4; KIf4, Rat gene coding for Klf4 protein; miRNA or miR, microRNA; mRNA, messenger RNA; qRT-PCR, quantitative real-time polymerase chain reaction. 


\section{Competing interests}

The authors declare no conflict of interest.

\section{Authors' contributions}

MAP conducted the molecular studies, immunoassays, and computational analyses, and drafted the manuscript. WMD and RW oversaw the one-yea carcinogenicity bioassay. HS participated in immunoassays, computational analyses, and miRNA isolation. DEW and EH participated in the experimental design, and helped to draft the manuscript. RHD conceived the study, supervised the work, and was responsible for manuscript edits. All authors read and approved the final version of the manuscript.

\section{Acknowledgements}

This study was supported by NIH grants CA090890, CA122959, CA90176, P30 ES00210, and a Pilot Project Grant awarded to MAP by the Environmental Health Sciences Center at Oregon State University. DNASTAR software was used under license from the Center for Genome Research and Biocomputing at Oregon State University.

\section{Author details}

${ }^{1}$ Linus Pauling Institute, Oregon State University, Corvallis, Oregon, USA. ${ }^{2}$ Department of Environmental and Molecular Toxicology, Oregon State University, Corvallis, Oregon, USA. ${ }^{3}$ School of Biological and Population Health Sciences, Oregon State University, Corvallis, Oregon, USA. ${ }^{4}$ R.H. Dashwood, 479 Linus Pauling Science Center, Oregon State University, Corvallis, OR 97331, USA.

Received: 29 July 2012 Accepted: 18 September 2012

Published: 24 September 2012

\section{References}

1. Parasramka MA, Ho E, Williams DE, Dashwood RH: MicroRNAs, diet, and cancer: new mechanistic insights on the epigenetic actions of phytochemicals. Mol Carcinog 2012, 51:213-230

2. Thomson JM, Newman M, Parker JS, Morin-Kensicki EM, Wright T, Hammond SM: Extensive post-transcriptional regulation of microRNAs and its implications for cancer. Genes Dev 2006, 20:2202-2207.

3. Lu J, Getz G, Miska EA, Alvarez-Saavedra E, Lamb J, Peck D, Sweet-Cordero A, Ebert BL, Mak RH, Ferrando AA, Downing JR, Jacks T, Horvitz HR, Golub TR: MicroRNA expression profiles classify human cancers. Nature 2005, 435:834-838.

4. Fu X, Han Y, Wu Y, Zhu X, Lu X, Mao F, Wang X, He X, Zhao Y: Prognostic role of microRNA-21 in various carcinomas: a systematic review and meta-analysis. Eur J Clin Invest 2011, 41:1245-1253.

5. Qi L, Bart J, Tan LP, Platteel I, Sluis T, Huitema S, Harms G, Fu L, Hollema H, Berg A: Expression of miR-21 and its targets (PTEN, PDCD4, TM1) in flat epithelial atypia of the breast in relation to ductal carcinoma in situ and invasive carcinoma. BMC Canc 2009, 9:163.

6. Liu N, Okamura K, Tyler DM, Phillips MD, Chung WJ, Lai EC: The evolution and functional diversification of animal microRNA genes. Cell Res 2008, 18:985-996.

7. Chen $\mathrm{K}$, Rajewsky N: The evolution of gene regulation by transcription factors and microRNAs. Nat Rev Genet 2007, 8:93-103.

8. Niwa R, Slack FJ: The evolution of animal microRNA function. Curr Opin Genet Dev 2007, 17:145-150.

9. Song G, Zhang $Y$, Wang L: MicroRNA-206 targets notch3, activates apoptosis, and inhibits tumor cell migration and focus formation. $J$ Biol Chem 2009, 284:31921-31927.

10. Adams BD, Cowee DM, White BA: The role of miR-206 in the epidermal growth factor (EGF) induced repression of estrogen receptor-a (ERa) signaling and a luminal phenotype in MCF-7 breast cancer cells. $\mathrm{Mol}$ Endocrinol 2009, 23:1215-1230.

11. Yan D, Dong XDE, Chen X, Wang L, Lu C, Wang J, Qu J, Tu L: MicroRNA-1/ 206 targets c-Met and inhibits rhabdomyosarcoma development. J Biol Chem 2009, 284:29596-29604.

12. Lin CC, Liu LZ, Addison JB, Wonderlin WF, Ivanov AV, Ruppert JM: A KLF4miRNA-206 autoregulatory feedback loop can promote or inhibit protein translation depending upon cell context. Mol Cell Biol 2011, 31:2513-2527.

13. Takahashi K, Yamanaka S: Induction of pluripotent stem cells from mouse embryonic and adult fibroblast cultures by defined factors. Cell 2006, 126:663-676.
14. Foster KW, Frost AR, McKie-Bell P, Lin CY, Engler JA, Grizzle WE, Ruppert JM: Increase of GKLF messenger RNA and protein expression during progression of breast cancer. Cancer Res 2000 60:6488-6495.

15. Huang CC, Liu Z, Li X, Bailey SK, Nail CD, Foster KW, Frost AR, Ruppert JM, Lobo-Ruppert SM: KLF4 and PCNA identify stages of tumor initiation in a conditional model of cutaneous squamous epithelial neoplasia. Canc Biol Ther 2005, 4:1401-1408.

16. Yang WT, Zheng PS: Krüppel-like factor 4 function as a tumor suppressor in cervical carcinoma. Cancer 2012, 118:3691-3702.

17. Zhao W, Hisamuddin IM, Nandan MO, Babbin BA, Lamb NE, Yang WW: Identification of Krüppel-like factor 4 as a potential tumor suppressor gene in colorectal cancer. Oncogene 2004, 23:395-402.

18. Ohnishi S, Ohnami S, Laub F, Aoki K, Suzuki K, Kanai Y, Haga K, Asaka $M$, Ramirez F, Yoshida T: Downregulation and growth inhibitory effect of epithelial-type Krüppel-like transcription factor KLF4, but not KLF5, in bladder cancer. Biochem Biophys Res Commun 2003, 308:251-256

19. Wang N, Liu ZH, Ding F, Wang XQ, Zhou CN, Wu M: Down-regulation of gut-enriched Krüppel-like factor expression in esophageal cancer. World J Gastroenterol 2002, 8:966-970.

20. Patel NV, Ghaleb AM, Nandan MO, Yang WW: Expression of the tumor suppressor Krüppel-like factor 4 as a prognostic predictor for colon cancer. Canc Epidemiol Biomarkers Prev 2010, 19:2631-2638.

21. Wang J, Place RF, Huang V, Wang X, Noonan EJ, Magyar CE, Huang J, Li LC: Prognostic value and function of KLF4 in prostate cancer: RNAa and vector-mediated overexpression identify KLF4 as an inhibitor of tumor cell growth and migration. Cancer Res 2010, 70:10182-10191.

22. Dang DT, Bachman KE, Mahatan CS, Dang LH, Giardiello FM, Yang VW: Decreased expression of the gut-enriched Krüppel-like factor gene in intestinal adenomas of multiple intestinal neoplasia mice and in colonic adenomas of familial adenomatous polyposis patients. FEBS Lett 2000, 476:203-207.

23. Shie $J$, Chen $Z Y, O^{\prime}$ Brien MJ, Pestell RG, Lee ME, Tseng CC: Role of gut-enriched Krüppel-like factor in colonic cell growth and differentiation. Am J Physiol Gastrointest Liver Physiol 2000, 279: G806-G814

24. Parasramka MA, Dashwood WM, Wang R, Abdelli A, Bailey GS, Williams DE, Ho E, Dashwood RH: MicroRNA profiling of carcinogeninduced rat colon tumors and the influence of dietary spinach. $\mathrm{Mol}$ Nutr Food Res 2012, 56:1259-1269.

25. Peltier HJ, Latham GJ: Normalization of microRNA expression levels in quantitative RT-PCR assays: identification of suitable reference RNA targets in normal and cancerous human solid tissues. RNA 2008, 14:844-852

26. Wang R, Löhr CV, Fischer K, Dashwood WM, Greenwood JA, Ho E, Williams DE, Ashktorab H, Dashwood MR, Dashwood RH: Epigenetic inactivation of endothelin-2 and endothelin-3 in colon cancer. Int J Cancer 2012, doi:10.1002/ijc.27762.

27. Evans PM, Liu C: Roles of Krüppel-like factor 4 in normal homeostasis, cancer and stem cells. Acta Biochim Biophys Sin (Shanghai) 2008, 40:554-564

28. Bernhardt M, Galach M, Novak D, Utikal J: Mediators of induced pluripotency and their role in cancer cells - current scientific knowledge and future perspectives. Biotechnol J 2012, 7:810-821.

29. Zhang N, Zhang J, Wang ZW, Zha L, Huang Z: Altered expression of Krüppel-like factor 4 and $\beta$-catenin in human gastric cancer. Oncol Lett 2012, 3:1017-1022

30. Wang $X$, Ling C, Bai $Y$, Zhao J: MicroRNA-206 is associated with invasion and metastasis of lung cancer. Anat Rec (Hoboken) 2011, 294:88-92.

31. Shie JL, Chen ZY, Fu M, Pestell RG, Tseng CC: Gut-enriched Krüppel-like factor represses cyclin D1 promoter activity through Sp1 motif. Nucleic Acids Res 2000, 28:2969-2976.

32. Chen JF, Mandel EM, Thomson JM, Wu Q, Callis TE, Hammond SM, Conlon FL, Wang DZ: The role of microRNA-1 and microRNA-133 in skeletal muscle proliferation and differentiation. Nat Genet 2006, 38:228-233.

33. Vickers MM, Bar J, Gorn-Hondermann I, Yarom N, Daneshmand M, Hanson JE, Addison CL, Asmis TR, Jonker DJ, Maroun J, Lorimer IA, Goss GD, Dimitroulakos J: Stage-dependent differential expression of microRNAs 
in colorectal cancer: potential role as markers of metastatic disease. Clin Exp Metastasis 2012, 29:123-132.

34. Shah MS, Schwartz SL, Zhao C, Davidson LA, Zhou B, Lupton JR, Ivanov I, Chapkin RS: Integrated microRNA and mRNA expression profiling in a rat colon carcinogenesis model: effect of a chemo-protective diet. Physiol Genomics 2011, 43:640-654.

doi:10.1186/1868-7083-4-16

Cite this article as: Parasramka et al:: A role for low-abundance miRNAs in colon cancer: the miR-206/Krüppel-like factor 4 (KLF4) axis. Clinical

Epigenetics 2012 4:16.

\section{Submit your next manuscript to BioMed Central and take full advantage of:}

- Convenient online submission

- Thorough peer review

- No space constraints or color figure charges

- Immediate publication on acceptance

- Inclusion in PubMed, CAS, Scopus and Google Scholar

- Research which is freely available for redistribution 